\title{
A Novel Semiautomatic Flesh Peeling and Seed Removing Mechanism for Dried Longan
}

\author{
Cheng-Han Li ${ }^{1}$, Yu-Ting Hsu ${ }^{1}$, Chun-Hung Hsieh ${ }^{2}$ and Ching-Wei Cheng ${ }^{3, *}$ \\ 1 Department of Bio-Industrial Mechatronics Engineering, National Chung Hsing University, \\ Taichung 402, Taiwan; cghan@smail.nchu.edu.com.tw (C.-H.L.); atin4100040063@gmail.com (Y.-T.H.) \\ 2 Department of Information Management, National Taichung University of Science and Technology, \\ Taichung 404, Taiwan; guu@nutc.edu.tw \\ 3 College of Intelligence, National Taichung University of Science and Technology, Taichung 404, Taiwan \\ * Correspondence: cwcheng@nutc.edu.tw; Tel.: +886-4-2219-5795
}

Citation: Li, C.-H.; Hsu, Y.-T.; Hsieh, C.-H.; Cheng, C.-W. A Novel

Semiautomatic Flesh Peeling and Seed Removing Mechanism for Dried Longan. Agriculture 2021, 11, 48. https://doi.org/10.3390/agriculture 11010048

Received: 10 December 2020 Accepted: 8 January 2021 Published: 10 January 2021

Publisher's Note: MDPI stays neutral with regard to jurisdictional clai$\mathrm{ms}$ in published maps and institutional affiliations.

Copyright: (C) 2021 by the authors. Licensee MDPI, Basel, Switzerland. This article is an open access article distributed under the terms and conditions of the Creative Commons Attribution (CC BY) license (https:// creativecommons.org/licenses/by/ $4.0 /)$.

\begin{abstract}
Longans, which are produced in tropical countries, are rich in nutrients and high in commercial value. To extend their shelf life, fresh longans are roasted to dry them out. For the convenience of cooking applications, the dried longan pulp is peeled and seeded beforehand. Presently, this is done manually; thus, this research aims to automate this process. A cutting tool with a serrated bending angle is used to cut the dried longan pulp, and then the longan is fixed by a lifting mechanism. The pulp is breached with a cutting tool and this breach is pushed up against a $6 \mathrm{~mm}$ hole in the silicone soft plate of the lifting mechanism to separate out the seed by squeezing. Commercially available dried longans are used in this experiment. The cutting surface width of the cutter designed in this study is $20 \mathrm{~mm}$, and the serration bending angle is $30^{\circ}$. This cutter operates at a speed of $29 \mathrm{~cm} / \mathrm{s}$ on dried longan pulp, attaining a success rate of $85 \%$. The lifting mechanism also presented in this study fixes the dried longan and uses a squeezing motion to separate the flesh from the seed with a silicone soft plate. Here, a lifting speed of $28 \mathrm{~mm} / \mathrm{s}$ achieves the highest rate of success at peeling, and the success rate of pulp-and-seed separation can reach $86.7 \%$.
\end{abstract}

Keywords: dried longan; lifting mechanism; silicone soft plate

\section{Introduction}

Longan (Dimocarpus longan Lour), an evergreen tree belonging to the Sapindus family, is grown as a commercial fruit crop [1,2]. Longan fruit is rich in nutrition and commercial value and is produced in tropical countries, especially Thailand and China [2,3]. Longan pulp can be used in traditional Chinese medicine to promote blood metabolism, relieve insomnia, and prevent amnesia because it contains many biologically active compounds and nutrients $[4,5]$. Taiwan is currently an important source of longan in Asia. The longan growing season is mostly concentrated in summer. Freshly harvested longan is usually high in temperature and moisture because of the weather, causing the quality of the fruit to deteriorate rapidly $[3,6]$. The freshness period is short, and the pulp will slowly ooze juice and deteriorate. This phenomenon is called pulp autolysis or pulp decomposition and greatly affects the pulp's quality, freshness, and value [7]. To extend its shelf life and facilitate storage, longan is usually processed. Typically, it is dried out through baking; dried longan can be stored for a longer time and made into canned food, wines, or jams. Separate baking processes are used for shelled and unshelled longan. Traditionally in Thailand, longan is first harvested, then shelled, and then baked [8]. The shelled longan is dried in hot air at $70{ }^{\circ} \mathrm{C}$ over $12-15 \mathrm{~h}$. The final moisture content of the longan can reach $22 \%$. The dried longan pulp will be golden in color [8,9]. It takes approximately $48-52 \mathrm{~h}$ to dry the shelled longan in hot air at $75^{\circ} \mathrm{C}$, and the final moisture content can be reduced to $22 \%$ [9]. In Taiwan, shelled longan is usually roasted directly through one of two methods: 
hot air baking or smoking. In each method, the dried longan will have a smoky fragrance and its pulp will appear dark brown after removing the shell.

Presently, the separation of the seeds and pulp of dried longan is still done manually by workers. The longan pulp contains high sugar content; after being dried in hot air, the pulp is caramelized and highly viscous. The shape of the fruit is irregular, complicating the process of seed removing. During manual seeding, the longan is typically heated beforehand. A knife is used to cut a hole in the pulp, and the seeds are removed. The operational process is slow and requires a lot of human resources. Because of changes in the rural population structure (particularly the aging of the population engaged in agriculture), human resources have been greatly reduced, and manual separation of the seed and the pulp may incur food hygiene and safety concerns. If machines can replace artificial longan deseeding operations, they can effectively reduce costs and improve production efficiency, food hygiene, lack of human resources, and other issues.

Longan-related research mostly focuses on its physical and chemical properties. Research on seeding machinery is quite rare, and there is no fully commercialized system available on the market. Xie et al. (2006) developed a seed removing machine for fresh shelled longan using air pressure as a power source to drive a pneumatic cylinder, which then drives a triangular blade to cut the freshly shelled longan together with its pulp [10]. The longan seeds are then pushed out by the pneumatic ejector bar to complete the work of removing them. The size of the cut for the fresh longan seeds will be affected by the thickness of the blade. If the cutting blade is too thick or too thin, it may cause the fresh longan cut to be incomplete, preventing the seeds from being removed. After the fresh longan is cut, the ejector bar's diameter and speed will also affect the seeding success and pulp loss rates. Pen et al. (2012) used simulated finger-drawing arcs to peel off dried longan flesh and a pneumatic cylinder as a power source to drive the triangular cam [11]. The cam controls the opening and closing of the L-shaped bracket, whose two ends are equipped with symmetrical pulp peeling arc blades. The two arc blades will cut a hole in the longan pulp for the seeds to be ejected using a rod ejector. The depth of occlusion between the two arc blades and the dried longan pulp, the opening and closing speed of the L-shaped bracket, and the pushing speed of the longan seeds' ejector bar will all affect the pulp peeling success rate.

This study aims to design a set of semiautomatic dried longan pulp peeling machines using a suitable cutter. The relationship between the shape and the speed of the cutter and the dried longan cutting success rate is also discussed. A lifting mechanism is designed using a silicone soft board to complete the seed removing operation. The dried longan is extruded through this board to peel the pulp and remove the seeds. The best speed and success rate of this process were found. The semiautomatic machine designed in this study can serve as a reference for future research on dried longan processing machinery.

\section{Materials and Methods}

\subsection{Dried Longan Sample}

Mature longan can be conical, heart-shaped, spherical, or other shapes. Some varieties have a significantly longer appearance on one side. After the drying process, the fruit's appearance will become wrinkled and rough. The dried longan's shape will be irregular, such that its flesh will be close to the inner core and appear approximately spherical or oval. Commercially available dried longan was used as our experimental material in this study. All experimental samples were stored under refrigeration. Before use, they were placed at room temperature to regain moisture, before being heated to $60.0^{\circ} \mathrm{C}$ in an oven to reduce the pulp's viscosity. The dried longan was used to test the flesh peeling and seed removing machine developed in this study. The average diameter of longan samples (pulp containing seeds) was $17.20 \mathrm{~mm}$ and that of dried longan seeds was $14.95 \mathrm{~mm}$. 


\subsection{Experimental Equipment}

The cutting tool of the dried longan pulp peeling machine is driven by a motor driver (VNH2SP30) and a direct current (DC) motor (JGB37-550) that has the maximum power of $60 \mathrm{~W}$. The lifting mechanism for the peeling of the pulp and removal of seeds is controlled by a stepping motor driver (AZD-CD, Oriental Motor Co., Ltd., Taiwan) and a stepping motor (AZM66AC-TS7.2, Oriental Motor Co., Ltd.). Once in position, the lifting mechanism is used to clamp, fix, and squeeze the dried longan, and a silicone soft board is used to peel the pulp and remove the seeds. The DC motor is controlled using an Arduino UNO controller. The positions of the stepping motor and lifting mechanism are controlled using a computer programming interface. The power consumption of the semiautomatic flesh peeling and seed removing machine is approximately $480 \mathrm{~W}$. The soft board is $50 \times 50 \mathrm{~mm}$ in size and is made of C-25 silicone (Asia Silicone Chemical Materials Co., Ltd., Taichung, Taiwan) with the tensile strength of $50 \mathrm{~kg} / \mathrm{cm}^{2}$, elongation of $750 \%$, and hardness of $25 \mathrm{HA}$.

\subsection{Semiautomatic Dried Longan Pulp Peeling Machine}

The semiautomatic dried longan pulp peeling and seed removing machine is based on aluminum extrusion. A stepping motor drives the eccentric shaft turntable in Figure 1a. The stepping motor's control can accurately clamp the dried longan. When the eccentric shaft turntable motor rotates upward, the dried longan-fixing jaws will open. At this time, the dried longan is placed on the pedestal. The eccentric shaft turntable is turned downward, and the jaws will clamp the dried longan. After the dried longan is fixed, the DC motor starts to drive the cutting tool's reciprocating link to cut the pulp. Because of the limited size of this machine, the longan pulp has been cut, and a silicone soft plate is installed along the aluminum extrusion groove, as shown in Figure 1a,b. To peel the pulp, the eccentric shaft turntable is turned up to the top, the dried longan in the holder is cut and squeezed with the silicone soft plate, and the longan seeds will pass through the hole. The longan pulp and seeds will then be separated; finally, the longan pulp remains under the silicone soft board, and its seeds will be placed on the top through the hole in the soft board, as shown in Figure 2.
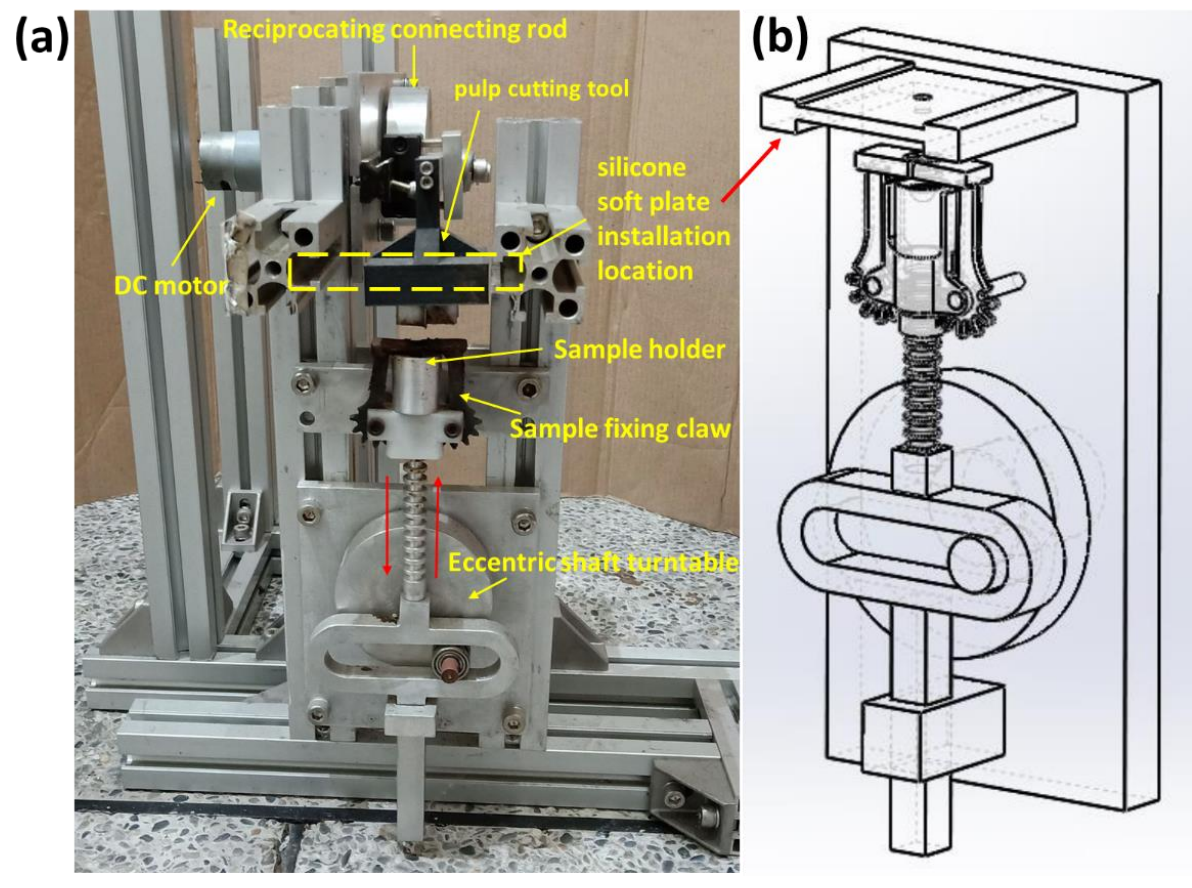

Figure 1. (a) Semiautomatic dried longan peeling machine. (b) Schematic of the installation position of the silicone soft board for peeling. 


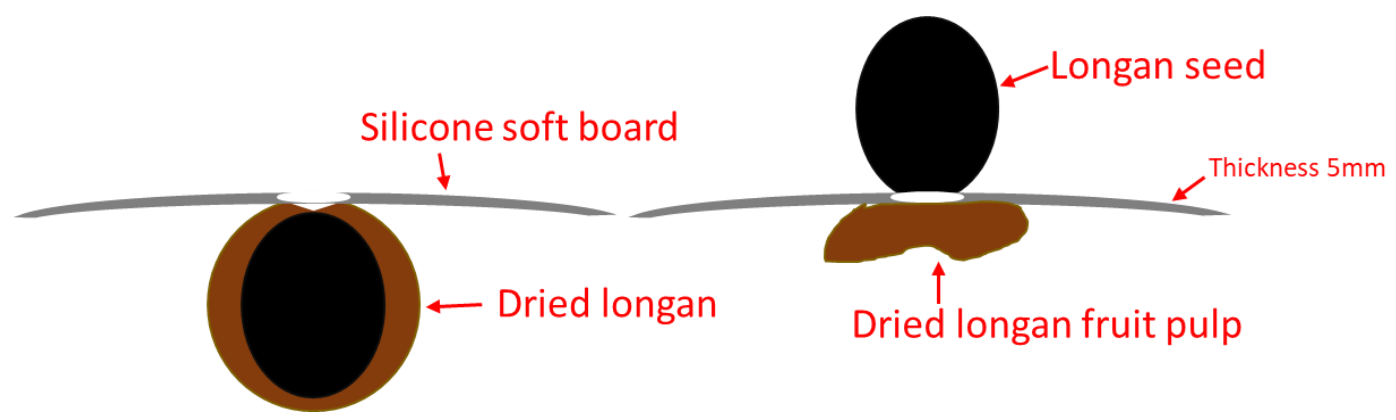

Figure 2. After the dried longan is cut, the hole cut in the pulp and the silicone soft board are squeezed against each other. The seeds and the flesh will separate; the pulp will stay under the silicone soft plate while the longan seeds will be extruded to the other side.

\subsection{Design of the Dried Longan Pulp Cutting Tool}

For manual longan peeling, the larger the hole in the pulp, the easier it is to remove the seeds. In this study, a longan cutting tool was designed, and the bending angle and width of the knife's cutting surface were changed, as shown in Figure 3. A knife drive mechanism was designed to perform a hole cutting test on the dried longan pulp. The formation and separation of chips in the cutting process are due to the material being subjected to the maximum shear stress; thus, we analyze it using shear-stress theory. When the tool and the cutting workpiece move relative to each other, the material is cut by shear force. This cutting surface is called a shear surface. For larger shear angles, the chip layer is thinner, resulting in smaller friction. The required cutting force is also smaller. By contrast, smaller shear angles make the chip layer thicker, resulting in larger friction. The cutting force is relatively large. Thus, this study designed a cutting tool for dried longan pulp.

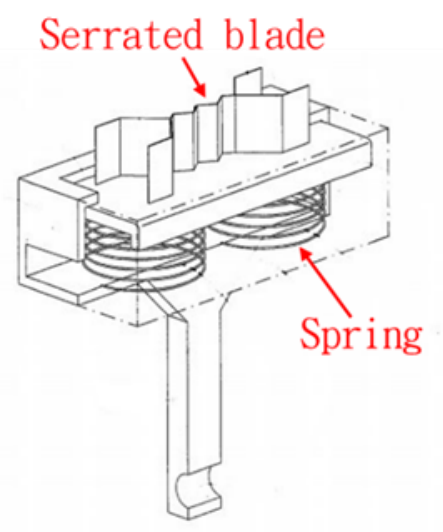

(a)

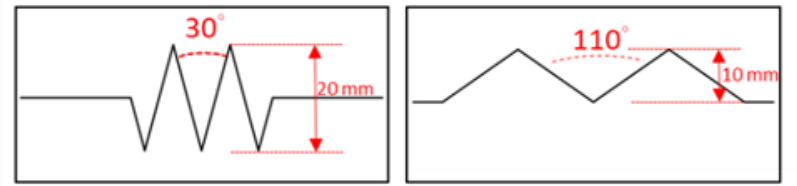

Figure 3. A schematic of a cutting tool for dried longan pulp. (a) A cutter with the blade width of $20 \mathrm{~mm}$ and $30^{\circ}$ serrated bending angle. (b) A cutter with the blade width of $10 \mathrm{~mm}$ and $110^{\circ}$ serrated bending angle.

Our cutting tool is made of a spring and aluminum foil, as shown in Figure 3. To prevent the longan seeds from being squeezed excessively by the cutter during the pressing process, the spring is used as an extrusion buffer and helps to make the knife edge fit more closely with the longan pulp. In this study, two types of knives were made for testing. For the first, the cutting surface width is $20 \mathrm{~mm}$, and the serration bending angle is $30^{\circ}$; for the second, the cutting surface width is $10 \mathrm{~mm}$, and the serration bending angle is $110^{\circ}$. Figure 3 shows the comparison of the two knives. At the same cutting speed, different knives' specifications are tested to find the most suitable for cutting dried longan pulp. 


\subsection{Design of the Cutting Tool's Driver Mechanism}

Cutter actuation adopts the scotch yoke mechanism, and a DC motor is used to convert circular motion into linear reciprocating motion. The linear reciprocating mechanism is designed to match the cutter-driven stroke shown in Figure 4. When the motor drives the disk to rotate, the shaft drives the rod to make a linear reciprocating motion to perform the opening operation. When the disk rotates through $0^{\circ}-180^{\circ}$, the driving rod moves forward and produces a maximum stroke of $45 \mathrm{~mm}$ at $180^{\circ}$; the rotation range is between $181^{\circ}$ and $360^{\circ}$, and the rod returns to the origin at $360^{\circ}$. The eccentricity of the eccentric shaft turntable is $34.3 \mathrm{~mm}$, and the maximum stroke of the cutter is $68.6 \mathrm{~mm}$.

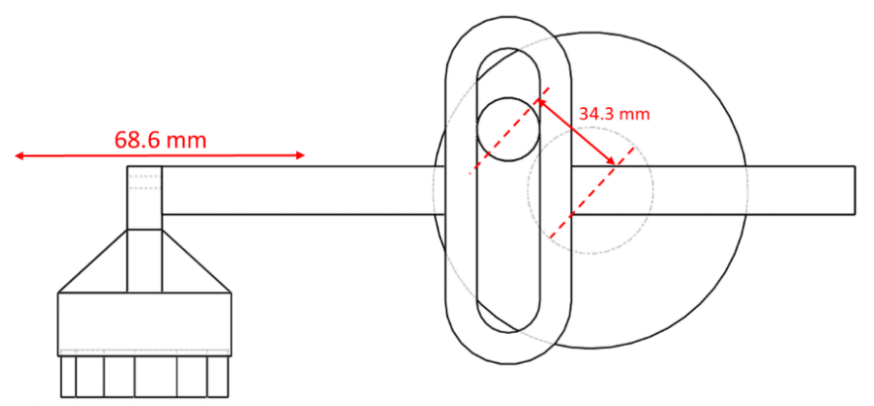

Figure 4. Dried longan pulp cutter reciprocating scotch yoke mechanism.

\subsection{Dried Longan Pulp Peeling and Seed Removing Mechanisms}

The pulp peeling and seed removing mechanisms are designed based on a wine opener's linkage mechanism. The main structure fixes the aluminum extruder on the aluminum plate. When the stepping motor rotates, it will drive the eccentric shaft disk. The eccentric shaft turntable will drive the row of teeth on the lifting mechanism to synchronously drive the ratchet to open and close the clamping arm. The eccentricity of the shaft on the turntable is $34.3 \mathrm{~mm}$, and the maximum displacement stroke is $68.6 \mathrm{~mm}$. There is a material holder above the lifting rod and a ball socket in this holder in which to place the dried longan. The side of the lifting rod is equipped with a row of teeth to drive the clamping arm. This arm's lower end has a semicircular ratchet for gear meshing transmission. The upper end of the clamping arm is equipped with clamping jaws to clamp and fix the dried longan. After the dried longan is clamped and fixed, a hole is cut in its pulp to remove its seed. Given that the dried longan's average height is approximately $16.8 \mathrm{~mm}$, the clamping height is set to $11.2 \mathrm{~mm}$ to stop the longan from turning during the opening process, as shown in Figure 5. Two-thirds of the dried longan's average length must pass through the silicone soft board to achieve good peeling. Considering that the thickness of the silicone soft board is $5 \mathrm{~mm}$, the mechanism's stroke is at least $16.2 \mathrm{~mm}$, and the total mechanical stroke of the peeling operation is at least $27.4 \mathrm{~mm}$, as shown in Figure 5 .

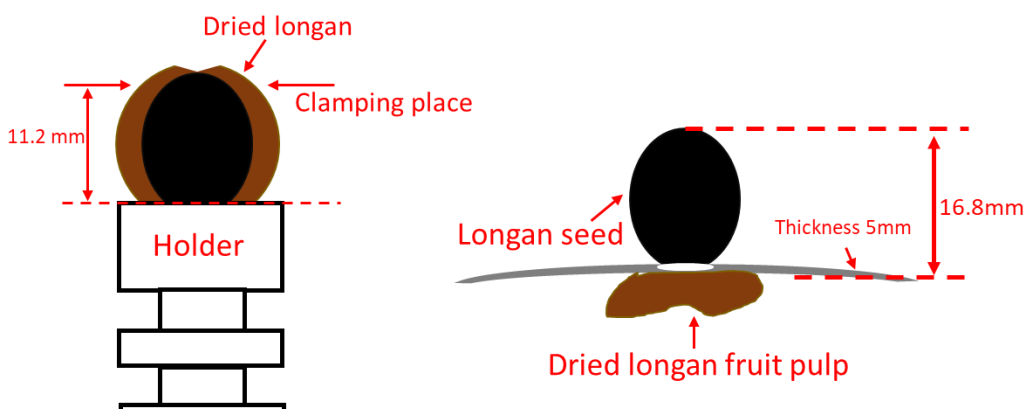

Figure 5. Schematic of the dried longan's clamping position. 
After the hole is cut, the eccentric shaft turntable is driven by the stepping motor, driving the lifting rod upward. Simultaneously, the row of teeth synchronously drives the clamping arm's ratchet wheel to rotate downward, the clamping arms open, and the material base is shifted upward. The dried longan in the material holder is lifted by the lifting mechanism. The average diameter of the dried longan is $17.2 \mathrm{~mm}$. The dried longan and the silicone soft plate are squeezed with a $6 \mathrm{~mm}$ through-hole silicone soft plate. This plate will deform, and its elasticity will be used to remove the dried longan pulp along the seed's surface. The longan seeds will be separated from the pulp through the hole in the plate and emerge on the other side, as shown in Figure 6.

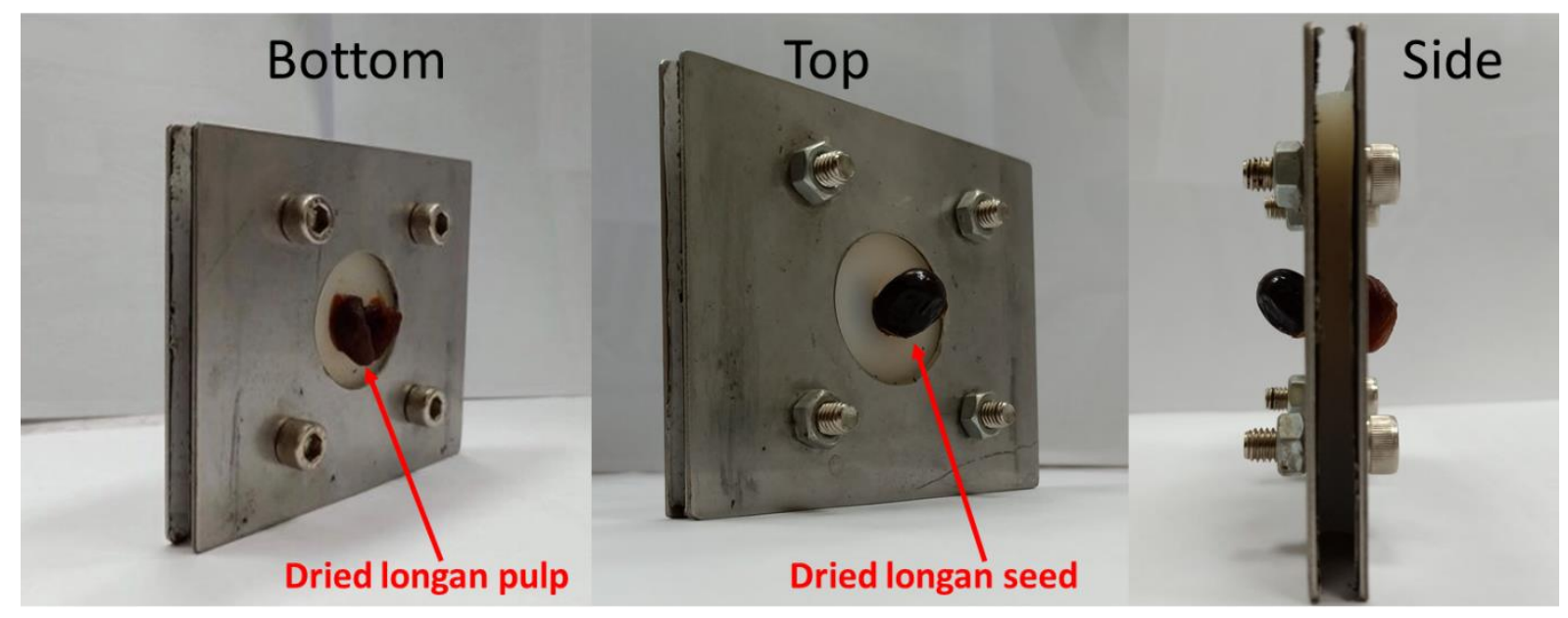

Figure 6. After squeezing the dried longan through the $6 \mathrm{~mm}$ hole of the silicone soft board, the pulp and seed are separated. The dried longan pulp will be below the plate while the seed will be above.

\section{Results and Discussion}

\subsection{Success Rate of Cutting with the Designed Machine}

Longan pulp cutting knives will affect hole size, affecting the success of subsequent peeling and seeding operations. In cutting the longan pulp, the dried seeds may be broken because of an uneven force applied by the cutter. The pulp also has a high sugar content, making it sticky. If the longan seeds are broken and shredded, they will stick to the pulp and seeding cannot be carried out. Figure 7 shows the definition of success and failure in this context. The hole cut in the dried longan pulp by the knife either (a) succeeds or (b) fails. If a hole is successfully cut in the dried longan pulp using the knife, the seed is clearly visible, as shown in Figure 7a; otherwise, it is not visible, as shown in Figure 7b.

Two different cutting tools were designed in this research, as described in Section 2.3 and shown in Figure 3. A DC motor drives the longan pulp cutting tool. This tool's speed is set to $508 \mathrm{~mm} / \mathrm{s}$, and 60 dried longans are used for the test. Table 1 shows the test results. The average width of the longan pulp breach formed by the cutter with a cutting surface width of $20 \mathrm{~mm}$ and a serrated bending angle of $30^{\circ}$ is $7.4 \mathrm{~mm}$; the success rate is $85 \%$, and the seed breakage rate is $11.7 \%$. For the cutter with a cutting surface width of $10 \mathrm{~mm}$ and a serrated bending angle of $110^{\circ}$, the average breach width is $5.5 \mathrm{~mm}$, the success rate is $63.3 \%$, and the seed breakage rate is $31.5 \%$.

The cutting range of the cutter with a $30^{\circ}$ serrated bending angle is larger than that of the one with a $110^{\circ}$ serrated bending angle. The former tool sticks easily to the pulp, but this does not affect the pulp opening operation. The $30^{\circ}$-serrated-bending-angle tool cuts holes with a high success rate and avoids breaking longan seeds. Experience with manual pulp peeling and seed removing shows that larger breaches in the pulp allow the smooth surface of the longan seeds to be clearly seen, enabling the pulp to be easily peeled and the seed to be removed. Therefore, the $30^{\circ}$-serrated knife was used in follow-up experiments. 
(a)

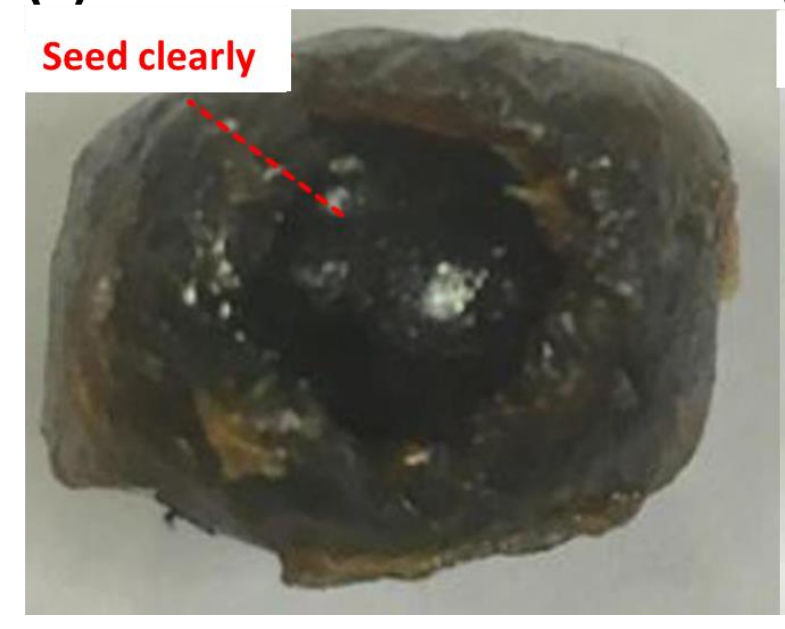

(b)

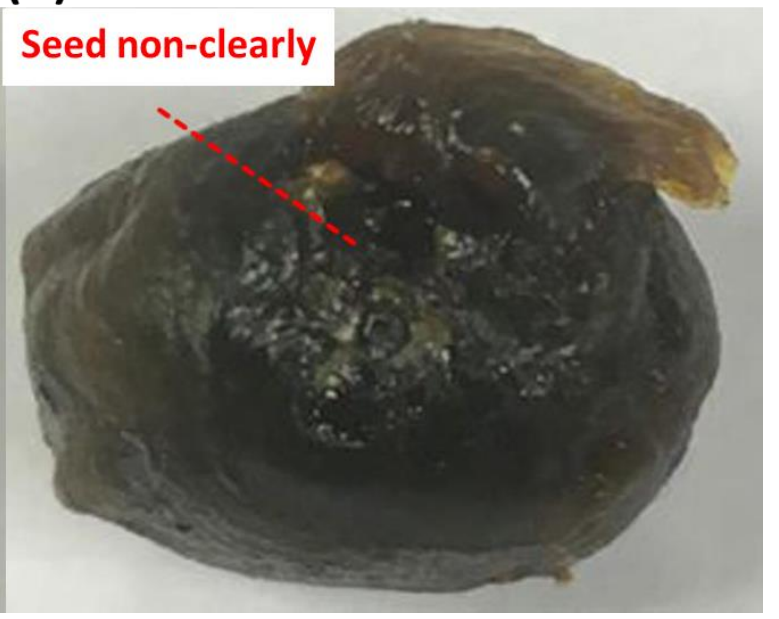

Figure 7. The hole cut in the dried longan pulp: (a) successfully; (b) fails.

Table 1. Cutting tool test results for dried longan.

\begin{tabular}{cccc}
\hline & Average Width of the Breach (mm) & Cutting Success Rate (\%) & Seed Damage Rate (\%) \\
\hline $\begin{array}{c}\text { Blade width is } 20 \mathrm{~mm} ; \\
\text { Serrated bending angle is } 30^{\circ}\end{array}$ & 7.4 & $85.0(51 / 60)$ & $11.7(7 / 60)$ \\
\hline $\begin{array}{c}\text { Blade width is } 10 \mathrm{~mm} ; \\
\text { Serrated bending angle is } 110^{\circ}\end{array}$ & 5.5 & $63.3(38 / 60)$ & $31.7(19 / 60)$ \\
\hline
\end{tabular}

\subsection{Success Rate of the Cutting Tool with Operating Speed}

The Arduino UNO controller adjusts the longan pulp cutter's speed through pulse width modulation (PWM) to adjust the DC motor's power supply voltage. The greater the PWM duty cycle, the faster the DC motor's speed and the greater the force of the cutter. A cutter with a width of $20 \mathrm{~mm}$ and a $30^{\circ}$ serrated bending angle was used to find the optimal cutting speed for dried longan pulp. The tests were carried out at speeds of 292.0, $330.0,380.0$ and $508.0 \mathrm{~mm} / \mathrm{s}$. Each speed was tested with 60 dried longans. Figure 8 shows the results.

When the cutting speed is $292.0 \mathrm{~mm} / \mathrm{s}$, the average width of the breach is approximately $7.5 \mathrm{~mm}$ and the success rate is approximately $95.0 \%(57 / 60)$. The test sample has no broken seeds. When the cutting speed is $330.0 \mathrm{~mm} / \mathrm{s}$, the breach's average width is approximately $7.3 \mathrm{~mm}$, the success rate is approximately $85.0 \%(51 / 60)$, and the seed breaking rate is approximately $10.0 \%(6 / 60)$. When the cutting speed is $380.0 \mathrm{~mm} / \mathrm{s}$, the average width of the breach is approximately $7.5 \mathrm{~mm}$, and the cutting success rate is approximately $80.0 \%$ (48/60), with a seed breakage rate of $15.0 \%(9 / 60)$. When the cutting speed is $508.0 \mathrm{~mm} / \mathrm{s}$, the average width of the breach is approximately $7.4 \mathrm{~mm}$, the success rate is approximately $85.0 \%$ (51/60), and the seed breakage rate is approximately $11.7 \%$ (7/60). The test results suggest that reducing the cutting speed helps to increase the cutting success rate and reduce the seed breakage rate. A cutting speed of $292.0 \mathrm{~mm} / \mathrm{s}$ yields the highest success rate without causing longan seed breakage.

\subsection{Test Analysis of Peeling Dried Longan Pulp}

A stepping motor is used to drive the lifting and clamping mechanism. The dried longan pulp is cut with a serrated cutter with a bending angle of $30^{\circ}$ at a speed of $292 \mathrm{~mm} / \mathrm{s}$. The lifting mechanism pushes the dried longan upward against the $6 \mathrm{~mm}$ hole in the C-25 silicone soft plate such that the seed is separated, as shown in Figure 2. 


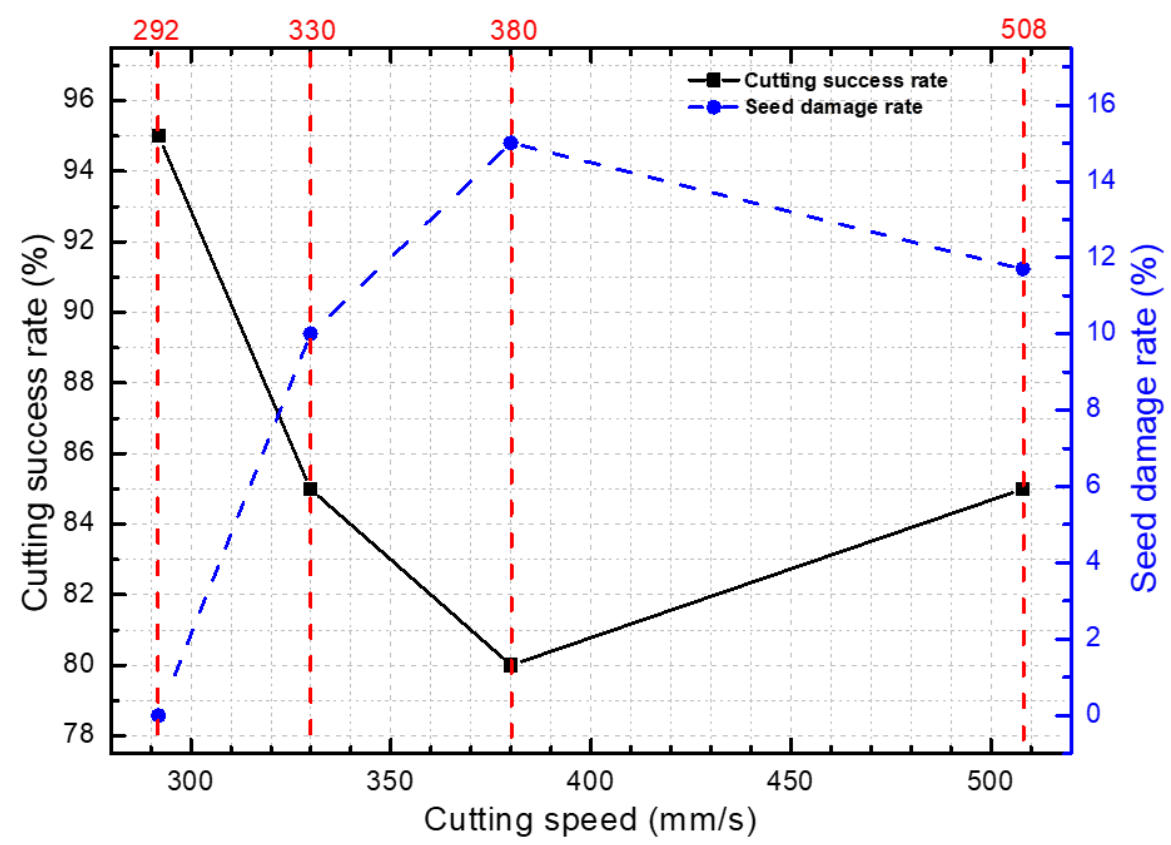

Figure 8. Success rates of the tool with a width of $20 \mathrm{~mm}$ and a sawtooth bending angle of $30^{\circ}$ at various cutting speeds.

The lifting mechanism's upward pushing speed is varied, and the relationship between this speed and the peeling success rate is analyzed. Tests are performed using 60 longans per group, and complete separation of the dried longan pulp and seed is defined as the successful peeling of the pulp. If the longan seeds have residual pulp, are broken due to pushing or cannot be separated from the pulp, the experiment is classified as a failure.

As shown in Figure 9, when the pushing speed is $70.0 \mathrm{~mm} / \mathrm{s}$, the peeling success rate is approximately $66.7 \%(40 / 60)$ and the failure rate is approximately $33.3 \%(20 / 60)$. The pulp peeling processes of 20 dried longans failed. Of these, 12 failed because pulp remained on the seeds, two failed because the seeds were broken, and six seeds did not smoothly pass through the silicone plate. When the pushing speed is $56.0 \mathrm{~mm} / \mathrm{s}$, the peeling success rate is $70.0 \%(42 / 60)$ and the failure rate is approximately $30.0 \%(18 / 60)$. Of the failures, 12 seeds had pulp remaining, two were broken, and four could not pass through the silicone soft board. When the pushing speed is $42.0 \mathrm{~mm} / \mathrm{s}$, the peeling success rate is $80.0 \%(48 / 60)$ and the failure rate is $20.0 \%(12 / 60)$. Of the failures, six seeds had pulp remaining, two were crushed, and four did not pass through the plate. For a pushing speed of $28.0 \mathrm{~mm} / \mathrm{s}$, the peeling success rate is approximately $86.7 \%(52 / 60)$ and the failure rate is $13.3 \%(8 / 60)$. Of the failures, four seeds had pulp remaining, two were broken, and two did not pass through the plate. For a pushing speed of $14.0 \mathrm{~mm} / \mathrm{s}$, the peeling success rate is approximately $86.7 \%(52 / 60)$ and the failure rate is approximately $13.3 \%(8 / 60)$. No samples failed because they did not pass through the soft silicone plate; however, six seeds had pulp remaining, and two were broken because of pushing.

The experiment shows that reducing the pushing speed will help to improve pulp peeling success rate. The dried longan may fall off the holder when passing through the plate if the lifting speed is too fast. Because there is not enough time for the seed to pass through the holes, some will fail to be removed. The dried longan peeling and seeding operation is equally successful at 28 and $14 \mathrm{~mm} / \mathrm{s}$; therefore, a speed of $28 \mathrm{~mm} / \mathrm{s}$ is preferable for efficiency.

Based on our results, we recommend using a tool with a cutting surface width of $20 \mathrm{~mm}$ and a sawtooth bending angle of $30^{\circ}$ to breach the pulp at a speed of $292.0 \mathrm{~mm} / \mathrm{s}$. After cutting, the dried longan should be squeezed with a silicone soft plate at a speed of $28 \mathrm{~mm} / \mathrm{s}$ for seed removing. Another 100 dried longan samples were prepared for testing 
to verify the mechanical stability. The peeling success rate was found to be $86.0 \%$, with a failure rate of $14 \%$. Of the failures, three seeds had pulp remaining and 11 seeds failed to pass through the plate. After the dried longan pulp was cut, if the dried longan seeds were broken or unable to be seen clearly, as shown in Figure 7, then it was judged that the cut had failed. After cutting, the dried longan and silicone soft plate could not pass through the through hole in the silicone soft plate, with residual pulp on the seed surface and dried longan seed breaks judged as failed peel, as shown in Table 2. The speed parameters of the semiautomatic flesh peeling in Table 2 and the seed removal mechanism of the dried longan are provided as references for future research.

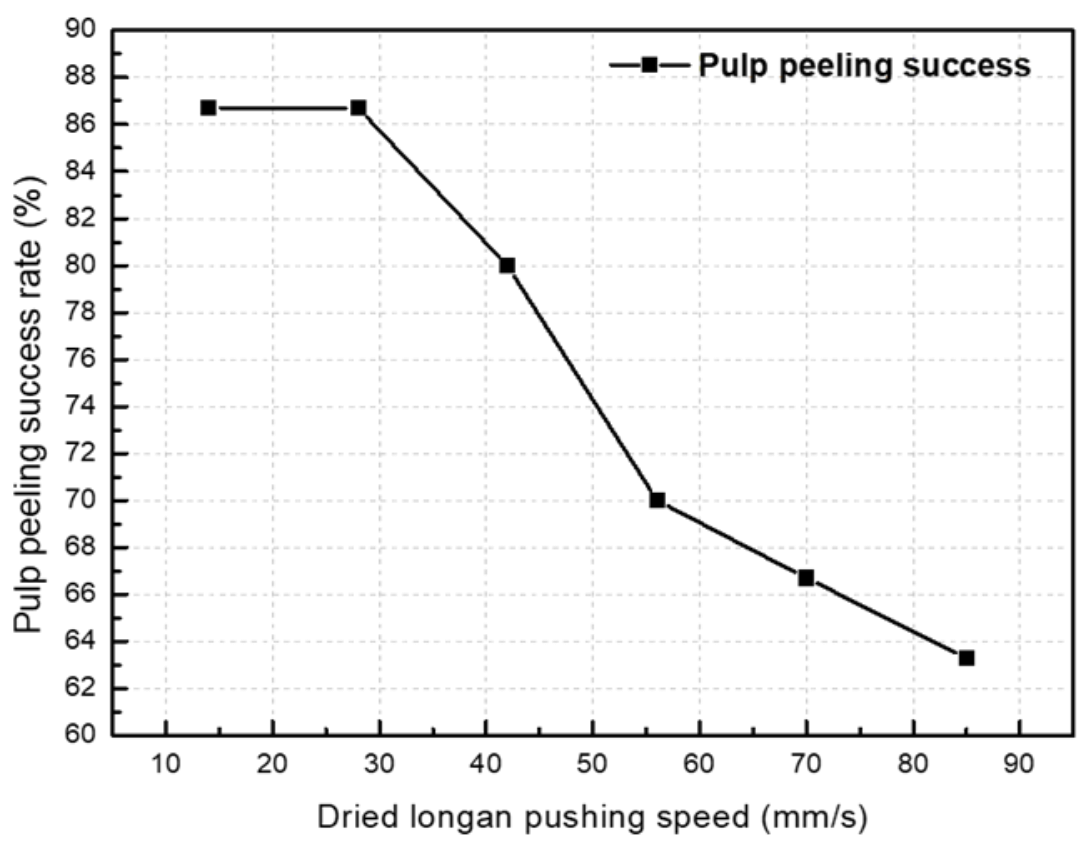

Figure 9. Pulp peeling success rate vs. pushing speed of the longan against the soft silicone plate.

Table 2. Parameters of the semiautomatic flesh peeling and seed removal mechanism for the dried longan design. Each parameter was tested using 60 dried longans.

\begin{tabular}{ccccccc}
\hline \multicolumn{7}{c}{ Blade width of $20 \mathrm{~mm}$ and sawtooth bending angle of $30^{\circ}$} \\
\hline Cutting speed (mm/s) & $\mathbf{2 9 2 . 0}$ & 330.0 & 380.0 & 508.0 \\
Cutting success rate (\%) & $\mathbf{9 5 . 0}$ & 85.0 & 80.0 & 85.0 \\
Seed nonclearly visible (\%) & $\mathbf{5 . 0}$ & 5.0 & 5.0 & 3.3 \\
Seed damaging rate (\%) & $\mathbf{0 . 0}$ & \multicolumn{7}{c}{10.0} & 15.0 & 11.7 \\
\hline The hole cut in the dried longan pulp and C-25 silicone soft plate peeling \\
\hline Dried longan pushing speed (mm/s) & 14.0 & $\mathbf{2 8 . 0}$ & 42.0 & 56.0 & 70.0 & 85.0 \\
Peeling success rate (\%) & 86.7 & $\mathbf{8 6 . 7}$ & 80 & 70 & 66.7 & 63.3 \\
Squeeze failure rate (\%) & 0.0 & $\mathbf{3 . 3}$ & 6.7 & 6.7 & 10.0 & 20.0 \\
Residual pulp on the seed surface (\%) & 10.0 & $\mathbf{6 . 7}$ & 10.0 & 20.0 & 20.0 & 16.7 \\
Seed damaging rate (\%) & 3.3 & $\mathbf{3 . 3}$ & 3.3 & 3.3 & 3.3 & 0.0 \\
\hline
\end{tabular}

Cutting speed of $292.0 \mathrm{~mm} / \mathrm{s}$ and pushing speed of $28 \mathrm{~mm} / \mathrm{s}$.

(100 dried longan samples)

Peeling success rate $(\%)$

Squeeze failure rate (\%)

86.0

Residual pulp on the seed surface (\%)

Seed damaging rate $(\%)$ 


\section{Conclusions}

This study shows that the use of serrated tools can increase the success rate of breaching dried longan pulp while decreasing the rate of seed breakage. The cutting tool's surface width is $20.0 \mathrm{~mm}$, and its bending angle is $30^{\circ}$. The use of a C-25 silicone soft plate with high elongation and high tensile strength can increase the success rate of pulp peeling and seed removing and extend the service lifetime of the pulp peeling parts. Reducing the cutting speed can increase the success rate. The optimal cutting speed is $292.0 \mathrm{~mm} / \mathrm{s}$, yielding a success rate of approximately $85 \%$, which is the best among the samples. Reducing the pushing speed helps to increase the success rate of pulp peeling and seed removing. The optimal pushing speed is $28.0 \mathrm{~mm} / \mathrm{s}$, yielding a success rate of approximately $86.7 \%$, which is the highest among the tests. Our pulp peeling mechanism was based on previous research by Pan et al. (2012) on squeezing down the thumbs of both hands [11]. The advantage of our mechanism is that the dried longan pulp cutting tool cannot be easily damaged and the overall structure is simple. Presently, if the peeling silicone soft plate is not manually installed in the machine, it takes around 5-6 s for each dried longan pulp to be cut and peeled, thereby showing that the average work efficiency of this design is lower than that of manual labor. Therefore, if this mechanical process can be converted into a workstation-based automatic pulp peeling and seed removal system, it is expected to be much faster and more hygienic than manual labor. The "Peeling and seed removal method" (patent number: TWI632863B) and "Pulp peeling device for dried longan" (patent number: TWM554709U) developed in the work have been patented in Taiwan.

\section{Patents}

We successfully obtained Taiwanese invention patents for the "Peeling and seed removing method" (patent number: TWI632863B) and the "Pulp peeling device for dried longan" (patent number: TWM554709U).

Author Contributions: C.-W.C. conceived and designed the experiments; Y.-T.H. performed the experiments; C.-H.L. and C.-W.C. wrote the paper; C.-H.L., C.-H.H. and C.-W.C. reviewed and edited the paper. All authors have read and agreed to the published version of the manuscript.

Funding: The authors thank the Agriculture and Food Agency, Council of Agriculture, Executive Yuan of the Republic of China for financially supporting, Taiwan, grant number 109AS-11.3.1-FD-Z1.

Acknowledgments: The authors thank the Agriculture and Food Agency, Council of Agriculture, Executive Yuan of the Republic of China for financially supporting.

Conflicts of Interest: The authors declare no conflict of interest.

\section{References}

1. Chen, Y.; Sun, J.; Lin, H.; Lin, M.; Lin, Y.; Wang, H.; Hung, Y.-C. Salicylic acid treatment suppresses Phomopsis longanae Chi-induced disease development of postharvest longan fruit by modulating membrane lipid metabolism. Postharvest Biol. Technol. 2020, 164, 111168. [CrossRef]

2. Jiang, Y.; Zhang, Z.; Joyce, D.C.; Ketsa, S. Postharvest biology and handling of longan fruit (Dimocarpus longan Lour.). Postharvest Biol. Technol. 2002, 26, 241-252. [CrossRef]

3. Lin, Y.; Lin, Y.; Lin, Y.; Lin, M.; Chen, Y.; Wang, H.; Lin, H. A novel chitosan alleviates pulp breakdown of harvested longan fruit by suppressing disassembly of cell wall polysaccharides. Carbohydr. Polym. 2019, 217, 126-134. [CrossRef] [PubMed]

4. Chen, Y.; Xie, H.; Tang, J.; Lin, M.; Hung, Y.-C.; Lin, H. Effects of acidic electrolyzed water treatment on storability, quality attributes and nutritive properties of longan fruit during storage. Food Chem. 2020, 320, 126641. [CrossRef] [PubMed]

5. Huang, F.; Hong, R.; Yi, Y.; Bai, Y.; Dong, L.; Jia, X.; Zhang, R.; Wang, G.; Zhang, M.; Wu, J. In vitro digestion and human gut microbiota fermentation of longan pulp polysaccharides as affected by Lactobacillus fermentum fermentation. Int. J. Biol. Macromol. 2020, 147, 363-368. [CrossRef] [PubMed]

6. Lin, Y.; Lin, Y.; Lin, H.; Shi, J.; Chen, Y.; Wang, H. Inhibitory effects of propyl gallate on membrane lipids metabolism and its relation to increasing storability of harvested longan fruit. Food Chem. 2017, 217, 133-138. [CrossRef] [PubMed]

7. Wang, D.; Zhang, H.; Wu, F.; Li, T.; Liang, Y.; Duan, X. Modification of Pectin and Hemicellulose Polysaccharides in Relation to Aril Breakdown of Harvested Longan Fruit. Int. J. Mol. Sci. 2013, 14, 23356-23368. [CrossRef] [PubMed]

8. Tippayawong, N.; Tantakitti, C.; Thavornun, S. Energy efficiency improvements in longan drying practice. Energy 2008, 33, 1137-1143. [CrossRef] 
9. Yang, K.-M.; Chiang, P.-Y. Effects of smoking process on the aroma characteristics and sensory qualities of dried longan. Food Chem. 2019, 287, 133-138. [CrossRef] [PubMed]

10. Xie, H.J.; Qing, Y.M.; Zheng, D.; Lin, C.F.; Li, Z.W. Design and experiment of a longan core-removing mechanism. Trans. CSAE 2006, 22, 211-213.

11. Pan, Y.T.; Lee, Y.N.; Chen, Y.H.; Cheng, C.W. The designation and test of dried longan pneumatic flesh-peeling machine. J. Agric. For. 2012, 61, 337-350. 